\title{
MODELING SECURITY RISK WITH THREE VIEWS
}

\author{
Susan J Lincke \\ Computer Science Department \\ University of Wisconsin-Parkside \\ 900 Wood Road \\ Kenosha, WI, USA \\ lincke@uwp.edu
}

\author{
Madhavi Adavi \\ Computer Information Systems \\ University of Wisconsin-Parkside \\ 900 Wood Road \\ Kenosha, WI, USA \\ himadhavi@gmail.com
}

\begin{abstract}
Organizations are responsible for implementing due care, or controls for risk, by calculating the likelihood multiplied by the impact for high-risk threats. Organizations cover their own risk expenditures and they do this independently. However, this may be myopic. We investigate a societal perspective by calculating risk via three models: an individual, organizational and societal view of security at a high level for two issues: ransomware and mobile privacy. For these two issues, we consider fault, responsibility, interdependency and ethics. By considering a more societal and interdependent solution, new or better solutions arise.
\end{abstract}

Keywords: Risk analysis, ransomware, mobile privacy, ethical risk, survey.

\section{INTRODUCTION}

Information security is problematic worldwide. Consider that in 2016, Symantec (2017) reports that $791,820,040$ data records were breached in the United States, which we note is over twice the population of the U.S. Add to that the rise in ransomware in 2016-18, and it could be argued that the criminals appear to be winning. This paper reevaluates risk in three ways to consider how to better defend against cybercrime.

In academic research, risk is discussed as threats and controls. Qualitative methods are used to present scenarios (or stories) to describe how a myriad of technologies could lead to risks, such as self-driving cars (Johnsen et al. 2017), artificial intelligence (Meek et al. 2016), decision support systems (Altman, Wood and Vayena 2018), net neutrality (Hartmann and Giles 2018), and privacy (Ahmad et al. 2016). Often they identify means or approaches to controls. Rarely are financial concerns discussed in technical research. Also, software development processes implementing risk analysis generally recommend qualitative or semi-quantitative (prioritized) analysis and not quantitative analysis (e.g., Gotterbarn and Clear 2004, CMMI 2010). Papers on game theory build economic analysis models but fall short of deriving actual financial numbers (Khalili 2018). Research that does estimate financial costs include rare cost of life papers (Kahn 1986).

In industry, however, financial or 'quantitative' risk analysis is commonly used, often in combination with qualitative techniques. Risk analysis in the corporation or organization asks: how much is our organization likely to lose if this threat occurs? Legally, corporations are responsible for doing risk analysis to ensure that they consider the amount of risk they are vulnerable and liable for. 'Due care' ensures that they implement controls up to this amount of level of vulnerability and liability. However, this liability is 


\section{Lincke and Adavi}

limited: organizations use cost-benefit analysis, which specifies that they need not spend more for controls than the estimated value of the risk (ISACA 2015). This risk is calculated by (1) from (ISACA 2013).

$$
\text { AnnualLossExpectancy }=\text { SingleLossExpectancy } * \text { AnnualRateOfOccurrence . }
$$

Risks are prioritized according to their potential Annual Loss Expectancy (ALE), which is the product of their single loss expectancy (SLE), which is the financial 'impact' of a single instance of the risk occurring, and the annual rate of occurrence (ARO), which is the likelihood or probability of the risk occurring. More accurately, each organization calculates risk according to its own self-interest, or to protect itself, via: ALE $[$ organization $]=S L E[$ organization $] * A R O[$ organization $]$.

The lack of quantitative risk analysis in research has drawbacks. Quantitative risk analysis can be a stronger motivation to act. It is the modus operandi in corporations, which means that academia could be more inline with and helpful to industry if we practiced quantitative risk analysis.

Another flaw is that the primary source of quantitative risk analysis is the corporation, which does it primarily for its own interest. In other words, it is not being calculated in technical research at the national or world level, and is never quantitatively calculated at the individual level. The implementation of regulation may be more a factor of lobbyists, news and public opinion than statistical reality. Technology research could provide expert opinions for quantitative risk analysts at the (inter)national, individual and organizational levels.

The goal of this paper is to lay out models for the three levels: individual, organizational, and societal, and perform a risk analysis for each level focused on ransomware and mobile application privacy. We evaluate these risks using a quantitative and qualitative risk perspective for each layer and recommend controls. Then we consider the effectiveness of the current models with a view toward problem resolution via fault, impact, responsibility, opportunities, knowledge and ethics. This raises opportunities to consider security at levels other than the organizational level.

\subsection{Individual-Level Risk Analysis}

The individual level of risk analysis is the average person. Quantitative risk analysis to us means: $A L E[$ individual $]=S L E[$ individual $] * A R O[$ individual $]$.

At the individual level, computer security risks may include that a computer become compromised, resulting in loss of financial information, lost files, and computer down time. Preventive technical controls generally include antivirus software (e.g., Norton: \$5-\$90/year, depending on volume) and disk backups. Procedural controls (for those of us with a low risk appetite) include careful behavior when opening email attachments, following web links, and when accessing personal/financial information. When our computer, regardless, becomes infected, a corrective control is a computer rebuild (e.g., Geek Squad: \$130 rebuild; \$200 with malware removal). Individuals are not regulated to protect themselves, and thus are free to make decisions about the use of preventive, detective, and corrective controls.

Unfortunately, relatively few individuals are security-aware. Many of us are aware of the risk likelihood of entering our credit information on a computer, and what we might lose (i.e., the risk impact). We also consider the cost of antivirus and computer rebuilds as justified defenses, considering the total risks that we may incur. For those with a very low risk tolerance or who have higher likelihood of identity theft (including a history of it), identity theft insurance (e.g., AllClearId.com: \$102/year) is also justifiable.

At the individual level, we do not do quantitative risk analysis to ensure we save money - but we also do seem to qualitatively factor in likelihoods and impacts in our decisions. From a self-centered perspective, it does make sense that one would not pay more for controls than intended losses. However, if this is true at the individual level, it certainly is justified at the organizational and societal levels as well. 


\section{Lincke and Adavi}

\subsection{Organizational-Level Risk Analysis}

Organizational-level risk analysis is the quantitative risk analysis applicable to corporations and other organizations. Currently, organizations are generally regulated to protect individuals' information, using preventive, detective and corrective controls. They do not calculate breach costs for their customers or employees, but instead to themselves, the organization. Legal requirements affects the SLE with a series of liability (L) costs as indicated in (2):

$$
\text { SLE }[\mathrm{Org}]=\text { Investigate }+ \text { Loss }+ \text { Recovery }+ \text { LostBusiness }+ \text { LegalL }+ \text { RegulatoryL }+ \text { ContractL }+ \text { EthicalL. (2) }
$$

\subsection{Societal-Level Risk Analysis}

The societal level can be thought of as the world or national level. Statistics here can indicate the status of controlling a world/national problem. We could consider that controls should be spent up until the cost of a problem: $A L E[$ nation $]=S L E[$ nation $] * A R O[$ nation $]$.

At the societal (or national) level, security reports indicate that cyber-crime is rampant, with some western nations reporting breaches annually and consistently at or near their population levels (e.g., Symantec 2017). Therefore, either there are insufficient controls (a spending problem) and/or we have an engineering problem, where security controls are insufficiently designed or are in the wrong place.

It is interesting that the national level implements preventive controls as regulation, and detective and corrective controls as law enforcement, courts, fines and prisons. The main preventive solution to solving cyber problems appear then to be at the organizational level, which is highly regulated.

This paper then considers two cases in the context of societal, organizational and individual risk analysis to consider real-world scenarios involving security problems. The two cases are ransomware and mobile privacy.

\section{THE CASE OF RANSOMWARE}

We will look at the three different perspectives: societal, organizational, and individual.

\subsection{Societal-Level Risk Analysis}

If we look at ransomware at the societal or global level, in $2016 \$ 850$ million was paid in ransom due to ransomware, affecting 30,000-50,000 devices monthly (Fortinet 2017). However, $\$ 850$ million dwarfs losses when considering the impact of business downtime: estimates for societal losses for small businesses may exceed $\$ 75$ billion annually (Fortinet 2017). Ransomware infections increased by 40\% in 2017 (Symantec 2018) and makes up well over 40\% of malware incidents in 2017 (Verizon 2018). These SLE numbers are estimated to happen yearly, and thus also serve as an ALE.

Law enforcement recommends not paying the ransom to discourage further criminal acts. Symantec (2017) reports that $47 \%$ who pay never get their files back. Ransomware is used not only to generate revenue, but also by nation-states as a decoy for stealing data, disrupting operations and disk wiping, e.g., via Petya/NotPetya, Phonywall, Disakil (Symantec 2018).

Ransomware is most usually spread via phishing attacks and web downloads at 83\% (Fortinet 2017), but has also spread via bots, worms, brute-force passwords and third-party app stores (Symantec 2017). Business models now include Ransomware-As-A-Service: e.g., Shark ransomware creators retain $20 \%$ of the take.

\subsection{Individual-Level Risk Analysis}

Ransomware scenarios include encrypted or wiped disks, but also locked out mobile phones. Consumers accounted for $69 \%$ of infections (Symantec 2017). Approximate ransoms included $\$ 679$ in 2016 (Fortinet 


\section{Lincke and Adavi}

2017) or \$522 in 2017 (Symantec 2018). These statistics serve as an SLE (if paid). Additional SLE costs include the inconvenience of computer downtime, potential loss of files and programs, and a computer rebuild (\$200). Ransomware is generally not personally threatening, unless spyware tracked certain transactions (financial, business) from the device. Unfortunately, most individuals are likely to learn about ransomware the first time they are attacked.

The main threat to ransomware is opening infected emails and visiting infect webpages. According to Symantec's 2018 Internet Security Threat Report, 1 in 412 emails contains malware (down from 113) and one in 13 web pages lead to malware. Using these statistics, we estimate that the likelihood (ARO) of being infected is (at least) annually, when not using controls.

Traditionally, cost-benefit analysis dictates that controls should be spent up to the ALE. Antivirus software can help, since Symantec $(2018,15)$ reports blocking around 1,242 ransomware attacks per day (excluding ransomware lookalikes). Other important controls include regularly backing up data, patching software, avoiding suspicious emails with attachments or web links, and avoiding macro-enabled Microsoft documents. Corrective controls include rebuilding the computer and changing passwords.

\subsection{Organizational-Level Risk Analysis}

Ransomware in business may corrupt individual computers or servers, or use doxing, where if the ransom is not paid, the information (e.g., medical) is released (Fortinet 2017). Server infections have increased to be the target of 30-37\% of ransomware attacks (Symantec 2018). KnowBe4 (2017) estimates that approximately one third of business over 1000 persons have dealt with ransomware in 2016, but smaller businesses encounter higher rates, to $88 \%$ for organizations with 1-50 employees. These statistics serve as ARO values. Businesses account for $31 \%$ of infections (Symantec 2017).

Businesses are prone to higher ransoms than individuals. Actual risk scenarios are provided by Fortinet (2017), where a medical center in California paid the equivalent of $\$ 17,000$ in bitcoin to restore their server data. Emery Brain Health Center was charged $\$ 180,000$ in ransom. A Kansas hospital paid an initial ransom, but when criminals demanded more money to restore data, the hospital refused. These serve as SLE values. Even if the ransom solution is selected, it may not succeed in restoring data.

At the organizational level, ransomware has additional troubling effects (Fortinet 2017):

- $63 \%$ encountered business-threatening downtime;

- $48 \%$ lost data or hardware;

- $34-42 \%$ paid a ransom;

- $34 \%$ lost money;

- $20 \%$ were forced to close their business;

- $3.5 \%$ indicate lives were put at risk.

The sum of these statistics indicate that the SLE include business downtime, estimated at 100 times the ransoms paid, to business closure (Fortinet 2017). This can be modeled as (3) or (4).

$$
\begin{aligned}
& S L E[\text { ransom }]=\text { BusCont }+ \text { Investigation }+ \text { Ransom }+ \text { Rebuild }, \\
& S L E[\text { noRansom }]=\text { BusCont + Investigation + Rebuild + Reload } .
\end{aligned}
$$

In both cases, manual operations are performed as a business continuity stage. An Investigation defines the type of corruption (encryption versus wipe), the spread of infection, and the determination of breach. The Rebuild reconfigures affected machines and ensures change of passwords.

In addition to recommended controls specified at the individual level, additional business controls include employees trained not to click on phishing email attachments or links; using spam filters; segmenting networks to secure data; and using advanced security techniques (Symantec 2017, Fortinet 2017).

Table 1 shows a summary of the quantitative risk analysis for ransomware, to the best of our knowledge. 
Table 1: Risk Analysis Summary of Ransomware.

\begin{tabular}{|c|c|c|c|c|}
\hline & Frequency & Impact & $\begin{array}{l}\text { Annual Loss } \\
\text { Expectancy }\end{array}$ & Severity \\
\hline Society & $\begin{array}{l}\text { Symantec }(201761) \\
\text { blocks at end of } \\
2016: \\
388,000 \text { attacks/day }\end{array}$ & Yearly & $\begin{array}{l}\$ 850 \text { million in } \\
\text { ransom; } \\
\$ 75 \text { billion total } \\
\text { (est) }\end{array}$ & \multirow{3}{*}{ 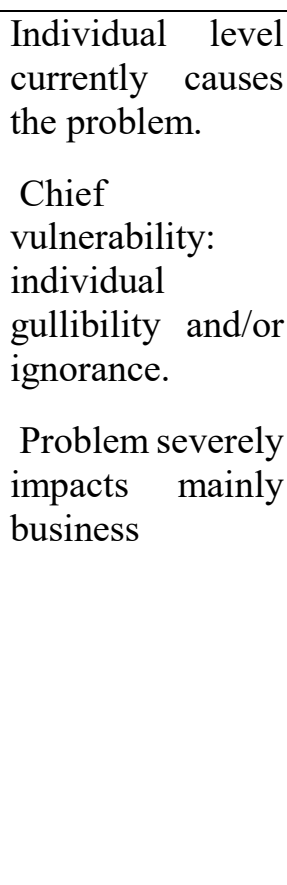 } \\
\hline Organization & $\begin{array}{l}33 \% \text { average; } \\
88 \% \text { for small org. }\end{array}$ & $\begin{array}{l}\$ 17,000-\$ 180,000 \\
\text { ransom } \\
\text { Lost business at } 100 x \text {; } \\
\text { potential life threatening; } \\
\text { investigation; rebuild } \\
\text { costs }\end{array}$ & $\begin{array}{l}\text { Ransom cost: } \\
\$ 5,610 \\
(33 \% * \$ 17,000)\end{array}$ & \\
\hline Individual & $\begin{array}{l}1 \text { in 412: Infected } \\
\text { email } \\
1 \text { in 13: Infected } \\
\text { web } \\
\begin{array}{l}\text { Assume } \quad A R O=1 \\
\text { year }\end{array}\end{array}$ & $\begin{array}{l}\$ 522 \text { as ransom; and } \\
\$ 200 \text { rebuild; } \\
\text { Nuisance: lost time and } \\
\text { files }\end{array}$ & $\begin{array}{l}\text { No controls: } \\
\$ 200-\$ 522\end{array}$ & \\
\hline
\end{tabular}

\section{THE CASE OF MOBILE PRIVACY}

This section on privacy is concerned with the divulging of private information as a result of the use of applications. Privacy by definition protects primarily at the individual level. Ahmad et al. $(2016,314)$ describe privacy as a personal control over what is shared, how much is shared, and how that information may be shared. They describe disclosure as the "knowledge of and control over how the disclosed information will be used by that entity". They point out that a list of Google or Yahoo searches can build a picture of personal habits, beliefs, sexual preferences, and other sensitive information. Thus, privacy has implications that affect both anonymity and free speech. To protect residents, Europe has a General Data Protection Regulation (GDPR) that defines requirements for privacy, including notice, purpose, consent, security, disclosure, access and accountability.

Since corporations and governments are made up of individuals, privacy also speaks to the interdependency that security affects; if an employee's computer is infected, that often leads to the infection of the organization.

\subsection{Societal-Level Risk Analysis}

Many in security recognize the risk of mobile spyware. Haley, Director of Symantec called mobile phones "great spying tools that can take pictures and record audio and video, and even report the location to an insider who could control the device" (Jaffee 2017, 23). Stafford, CEO Diligent, points out common information captured on cell phones, including "contacts, calendars, geolocations, photos, attachments and more" (Hoffman 2017, 26). Gates questions why many Google Play Store apps need access to contacts, location, and may modify or delete SIM card contents (Hoffman 2017). Michelsen, from Zimperium, said that these 'imposter applications' are "loaded with spyware, remote access Trojans and bots that gave cybercriminals complete control over users' mobile devices" (Hoffman 2017, 26). The McAfee Mobile 


\section{Lincke and Adavi}

Threat Report (2018a, 3) says: "The Google Play store is under siege. What has changed over the years is the growth in the number of infected devices, which now typically can reach into the millions as we discover new aggressive campaigns." Prime risks affecting both business and individuals include gaining email access, which enables an attacker to reset passwords and access numerous online services.

Symantec (2018) calls applications 'grayware' that host "hack tools, accessware, spyware, adware, dialers, and joke programs." They report that $63 \%$ of grayware apps leak the phone number, and $37 \%$ leak the location. Only $20 \%$ of Android phones are patched to the safest O.S. version.

We observed one such mobile scenario. In monitoring an application on an Android smart phone via a protocol analyzer in January 2017, the phone's other applications, IMEI (phone identification) and versions were uploaded to the developer organization. (See Figure 1.) It is not our goal to single out the vendor of this application, since any app could obtain this information. However, it is worth noting that this application was developed in a foreign country that is known either for its cyber-espionage or cyber-crime. It is possible that the vendor could sell this information to a crime syndicate or government - or the information may be used for legitimate purposes. The reviews of this application indicated that users were not aware of any possible security issues with the application.

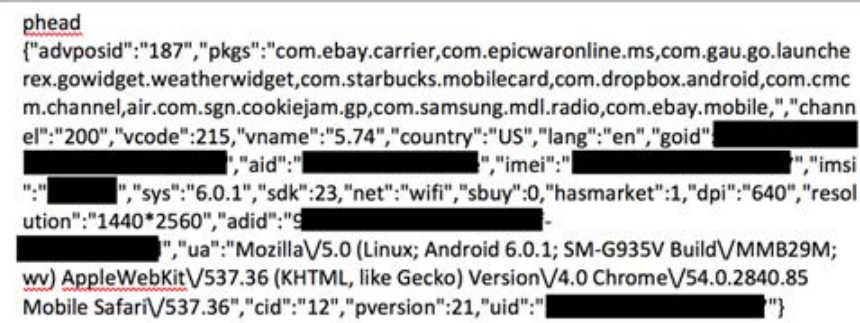

Figure 1: Example Uploaded Information from Android App.

We observed the permissions various weather apps require to operate in a phone, through searching an Android App Store under the Permissions tab. (See Table 2, taken March 2019). Apps can access a wide variety of information, with little information as to the magnitude of the information obtained. Even with this list it is difficult to make an informed decision of whether an app is accessing information it should not access. Some applications (if you fully read the manifest) enable access to:

- The user's phone identity (IMEI) and/or call metadata. The IMEI may be used to identify reliable app contributions. However, it may include phone numbers of calls received and length of each call, enabling the building of a huge network diagram showing who is connected to whom. This is the same metadata that the US government accessed without oversight.

- Their list of bookmarks and browser history. This can be used for spear phishing, blackmail, and pharming.

- The complete list of applications installed on the user's device.

There are both legitimate and nefarious reasons for obtaining the complete list of applications: either to learn the interactions an app is encountering - or to apply zero day attacks addressing certain applications, or learn media tendencies for spear phishing. In addition, from such information it is possible to learn where people work, which banks they use, and which home automation devices they use. Any of these are more easily attacked with such information. 
Table 2: Access for Weather Apps.

\begin{tabular}{|c|c|c|c|c|c|c|c|}
\hline Permission List & $\begin{array}{c}\text { MSN } \\
\text { Weather }\end{array}$ & $\begin{array}{l}\text { Weather } \\
\text { Channel }\end{array}$ & $\begin{array}{l}\text { Weather } \\
\text { View }\end{array}$ & $\begin{array}{l}\text { Rain } \\
\text { Alarm }\end{array}$ & $\begin{array}{l}\text { Weather } \\
\text { Bug }\end{array}$ & $\begin{array}{c}\text { Accu } \\
\text { Weather }\end{array}$ & $\begin{array}{c}\text { Go } \\
\text { Weather }\end{array}$ \\
\hline Use your location & 1 & 1 & 1 & 1 & 1 & 1 & 1 \\
\hline Full network access & 1 & 1 & 1 & 1 & 1 & 1 & 1 \\
\hline $\begin{array}{l}\text { Create accounts and set } \\
\text { passwords }\end{array}$ & 1 & & & & & & \\
\hline View network connections & 1 & 1 & 1 & 1 & 1 & 1 & 1 \\
\hline Use your USB media & 1 & & 1 & 1 & 1 & 1 & 1 \\
\hline $\begin{array}{l}\text { Read calendar events plus } \\
\text { confidential information }\end{array}$ & & 1 & & & & & \\
\hline $\begin{array}{l}\text { Read phone status \& } \\
\text { identity }\end{array}$ & 1 & & 1 & & 1 & & 1 \\
\hline User your microphone & & & & & & 1 & \\
\hline Retrieve running apps & & & & & & & 1 \\
\hline Modify system settings & & & & & & & 1 \\
\hline Read Google service config. & & 1 & 1 & 1 & 1 & 1 & 1 \\
\hline $\begin{array}{l}\text { Access your accounts } \\
\text { (identity and/or contacts) }\end{array}$ & 1 & 1 & & & & & 1 \\
\hline Run at startup & & 1 & 1 & 1 & 1 & 1 & 1 \\
\hline Use Bluetooth and/or WiFi & 1 & 1 & 1 & 1 & 1 & 1 & 1 \\
\hline Disable screen lock & & & & & & 1 & 1 \\
\hline \begin{tabular}{|lll}
$\begin{array}{l}\text { Prevent } \\
\text { sleeping }\end{array}$ & device & from \\
\end{tabular} & & 1 & 1 & 1 & 1 & 1 & 1 \\
\hline $\begin{array}{llll}\text { Access } & \text { Device } & \& & \text { App } \\
\text { History } & & & \\
\end{array}$ & 1 & & & & & & \\
\hline Change audio settings & & & & & 1 & & \\
\hline
\end{tabular}

\subsection{Individual-Level Risk Analysis}

At the individual level, many have grown to accept that our location and interests are sold to solicit advertising. However, increased risks of spear phishing and zero-day attacks resulting in ransomware, blackmail, bot software, or bank attacks is not acceptable. McAfee Mobile Threat Report (2018a) reports governments are using spyware to monitor and close down activists in their country.

At the individual level it is very difficult for even security experts to determine whether an app is safe or not. The McAfee Mobile Threat Report (2018a, 3) indicates that $23 \%$ of mobile malware includes spyware 


\section{Lincke and Adavi}

and McAfee's Quarterly Threats Report $(2018 \mathrm{~b}, 11)$ indicates that 8-13\% of mobiles reported infections between 2017-2018. Thus the ARO of being infected with spyware is $23 \%$ times 8 to $13 \%$, or $2-3 \%$.

One preventive control is to be careful in purchasing apps, even in safer app stores. When we searched though weather apps at the Google App store, we found differences in the types and quantity of permissions the app was requiring. Should your weather app "read calendar events plus confidential information" or "add or remove (Identity) accounts" or read your contacts, for example?

Another preventive control in addition to anti-virus software is the selection and configuration of the operating system. Although iOS has a similar number of vulnerabilities to Android (Symantec 2017), the iOS operating system allows the user to turn off app access to questionable features, and newer versions of Android are adding similar features. Other operating systems including Windows and UNIX would also benefit by implementing such features.

\subsection{Organizational-Level Risk Analysis}

Apps and devices which may sell such information pose a greater threat to many organizations. The potential sale of individual information to crime syndicates or foreign governments can result in cybercrime or cyber-espionage that can put the organization itself at risk. Thus, organizations appear to be more at risk from personal device security, even than individuals.

One risk concern is an organizational breach. The Ponemon's 2018 Cost of Data Breach Study provides the best SLE estimates for security breach costs. The average cost of a breach is $\$ 3.86$ million (\$7.91 million U.S.), with an average per record cost of $\$ 157$ (\$258 U.S.) for malicious or criminal attacks. The churn rate is $3.4 \%$ (3.6\% U.S.) The full likelihood (ARO) of breach (of which mobile data is only a part) is $27.9 \%$ (26.9\% U.S.) over 24 months (Ponemon 2018). Alternatively, the ARO can be calculated as the sum of risk of unprotected mobile devices in the organization's network. However, the SLE costs do not include potential costs arising from espionage that can threaten companies and nations. Table 3 shows a summary of the quantitative and qualitative risk analysis for mobile privacy, to the best of our knowledge.

Table 3: Risk Analysis Summary of Mobile Privacy.

\begin{tabular}{|c|c|c|c|c|}
\hline & Frequency & Impact & $\begin{array}{l}\text { Annual Loss } \\
\text { Expectancy }\end{array}$ & Severity \\
\hline Society & $\begin{array}{l}\text { Kaspersky } \\
\text { (2017) found: } \\
1.5 \text { M malicious } \\
\text { mobile installation } \\
\text { packages in Q3 } 2016\end{array}$ & Yearly & Unknown & \multirow{3}{*}{$\begin{array}{l}\text { Basic threats: } \\
\text { Malware download } \\
\text { at individual level; } \\
\text { spyware/phishing } \\
\text { attacks at business } \\
\text { level. } \\
\text { Chief vulnerability: } \\
\text { individual gullibility } \\
\text { and/or ignorance. } \\
\text { Problem impacts } \\
\text { mainly individuals } \\
\text { and organizations } \\
\text { vulnerable to spying. }\end{array}$} \\
\hline $\begin{array}{l}\text { Organiz- } \\
\text { ation }\end{array}$ & $\begin{array}{l}\begin{array}{l}2-3 \% \text { per mobile } \\
\text { device OR }\end{array} \\
13.95 \% \text { per org. }\end{array}$ & $\begin{array}{l}\text { Breach costs: } \$ 157 \text { per } \\
\text { record; } 3.4 \% \text { churn } \\
\text { rate. } \\
\text { Lost proprietary info. }\end{array}$ & $\begin{array}{l}\text { Avg. } \quad \text { breach: } \\
\$ 3.86 \quad \text { Million } \\
* 13.95 \%\end{array}$ & \\
\hline Individual & $\begin{array}{l}2-3 \% \text { per mobile } \\
\text { device; } \\
\text { In many countries: } \\
1-2 \text { times per } \\
\text { individual per year }\end{array}$ & $\begin{array}{l}\text { \$200 to rebuild; } \\
\text { Nuisance } \\
\begin{array}{l}\text { devastating: to } \\
\text { criminal false } \\
\text { bankruptcy }\end{array}\end{array}$ & $\$ 200+$ & \\
\hline
\end{tabular}




\section{Lincke and Adavi}

Recommended corporate security for mobile devices include: security training (purchase from primary app stores, limit device permissions), separation of personal and professional accounts, archived email, whitelisted applications, mobile device management and mobile application management enterprise solutions (Jaffee 2017, Hoffman 2017, Symantec 2018).

\section{ANALYSIS}

The societal level is a good indicator of how a world problem is faring. The earth has eradicated polio, typhoid, and scarlet fever, but not cyber-security threats. (Note that disease immunization is required at the individual level via regulation or offered freely.) At the societal level, each entity (organization, individual, nation) appears to be fighting security via financing and building their own security controls. However, it can be argued that this is not working: crime pays and will continue to. Furthermore, at the societal level, we see that governments have a hard time defending against security crimes, since the Internet is borderless and jurisdiction is an issue.

\subsection{Individual Level Risk Analysis}

The chief vulnerability against ransomware and privacy attacks is opening malicious emails, clicking on infected links, and downloading Trojan spyware apps. A quantitative risk analysis definitely justifies the purchase of antivirus software (versus frequent rebuilds), but unfortunately antivirus software is not foolproof. The other required control is being careful in opening emails and accessing web pages. One main problem is that much of society is not security savvy, including the individual level and small businesses. This manual control requires excellent knowledge and savvy (if not intuitive) guessing skills. Small businesses are actually threatened by malware, such as ransomware.

One option is to train every person on earth to be fearful of opening attachments or following links. This seems undoable and undesirable. However, to change this means that we need an engineering solution that does not rely on savvy computer users, and that automates security violation reporting. This would result in a societal interdependence of how risk is solved, since changing the individual level also affects the organizational and societal levels.

Computer-savvy and less-savvy people need options to control their own computers, and offer input to antivirus companies and software manufacturers via a network of contributions. In effect, all operating systems would implement a jail implementation for all applications, which is a security mechanism for quarantining untrusted running programs. This would follow the lead of iOS, but be implemented with all operating systems, including Windows, UNIX, and Android. This would move the main security control from a guessing game to a detection mechanism, with automated reporting of inappropriate accesses to software (e.g., antivirus, OS) companies as well as the user.

All operating systems (or other software) could limit API capability and provide users the ability to configure these options:

- Show icons, prompts, or LEDs indicating the active use of microphone and camera;

- Display programs currently reading or modifying the file system (including O.S), to fight ransomware and exfiltration;

- Display programs currently transmitting, including the volume of traffic sent and received, to determine appropriateness of those programs' access;

- Restrict access of applications to privacy data, such as website information, personal email contacts, personal directory files, with just-in-time override capability;

- Configure the appropriateness of each application to access specific rights;

- Monitor the configuration of program access rights;

- Centrally report when unknown applications appear to be exceeding boundaries;

- Easily locate and uninstall or hinder rogue program execution; 
- Potentially display encrypted data in transmitted data and cookies before they are encrypted, to catch exfiltration data (This is the most risky of all proposals, and if implemented, would be allowed only at the administrator level).

This technique would enable the sharing of information on rogue applications to users, and enable them to share information on rogue applications to antivirus, OS and app vendors automatically or manually. Understandably, there will be issues with such implementations, but this general direction could help in fighting cyber-crime considerably. Individuals and organizations purchase products and can vote with their pocketbooks for systems that enable them to be informed and enabled.

\subsection{Organizational Level Risk Analysis}

The only level that may be security savvy and responsible is at the larger organizational level - and even here, knowledge, controls and spending appear inadequate. Even when security is taken seriously and spending is adequate, these organizations are proving penetrable. Thus, even here security controls appear to be insufficiently designed, particularly related to the human 'firewall' of people. These organizations too would benefit from tighter security monitoring and reporting, as mentioned in the previous section.

A second issue is our expectation that the organizational level will solve our societal security problems, since their motivation is self-preservation and not societal-preservation. This research has shown that organizations are primarily susceptible to individual-level lack of security. Instead of evaluating security from a holistic perspective, the self-centered approach focuses on training to avoid phishing and malware. An interdependent approach would consider problems across different layers.

If organizations analyzed beyond ALE[organization] to also consider ALE[society], there is a chance for development of novel security-oriented products, such as this or other proposals, resulting in higher sales, due to volume or price - or to find other low-cost ways of protecting stakeholder security. Since they are unlikely to go this route, research can perform this analysis for them and us.

\subsection{Societal Level Risk Analysis}

The toolbox at the national level is generally regulation. The European Union has made great strides with GDPR. This regulation should have an effect of restricting spyware but also increasing security expenditures at the organizational level.

Lacking or even with such regulation, the advantage of societal interdependence and cooperation means that individuals should be able to view and report abuse of O.S. features, thereby signaling problems to antivirus, software manufacturers, law enforcement, and/or app stores. Software and app stores are then strengthened for security. This helps governments and corporations fight cyber-espionage and cyber-crime. It also helps organizations and individuals fight ransomware and other malware. Alternatively, when app stores (or third party verifiers) detect abuse of privilege, this also helps fight rogue software, enabling more sales for legitimate app developers. This also protects individuals and organizations alike.

Fortinet writes in their security report $(2017,9)$ that an army of coordinated effort is required: "Real-time actionable intelligence must be shared between the different security layers (and generally vendor products) and even extended to the broader cybersecurity community outside of your organization such as Computer Emergency Response Teams (CERTs), Information Sharing and Analysis Centers (ISACs), and industry coalitions like the Cyber Threat Alliance."

From a research perspective, analyzing risk by evaluating all three layers enables us to see problems at a higher level, enabling us to understand why the problems are occurring, and offer alternatives for where the problem may best be solved. The implementation may be complex and require a coordinated interorganization approach, but a coordinated approach can leverage financial pressure to solve problems in a more effective way. 
An issue with this model for research is that statistics are temporary, varying from year to year; research prefers a lasting solution. However, advantages include: 1) this research works with business where they are; 2) these results are a picture of reality in time (even if temporary); and 3) we benefit governments, individuals and organizations simultaneously. Finally, statistics can be updated since references are provided.

\section{CONCLUSION}

We have demonstrated risk assessments from different perspectives, with a focus of mobile privacy and ransomware. Considering just these two areas, current human firewall systems are inadequate. However, individuals may be empowered through advanced security notifications and configurations in the operating system. A network of information could then help inform O.S. and antivirus manufacturers of rogue software in an interdependent way. This enables all levels to benefit by improving security at the individual level.

Viewing security at the societal, organizational and individual levels helps us to understand and approach solutions where they should be solved. The societal view currently demonstrates that cyber-crime pays. Individual computer users usually lack security savvy, and even knowledgeable users are somewhat defenseless against nefarious software. Organizations have the best level of controls. However, their current self-centered approach does not enable organizations to recognize their interdependence, nor see opportunities for collaboration or to develop novel security solutions. Research needs to recognize that organizations are financially motivated, and provide a more holistic view. Research may also best fill the gap to assess societal and individual risk analysis perspectives.

\section{REFERENCES}

Ahmad, A, M. Preeti, M, F. Ono, C. Priest, J. Suarez, and A. Urcioli. 2016. "The Information Privacy Domain”. International Journal of Information Privacy, Security and Integrity vol. 2, no. 4, pp. 307329.

Altman, Micah, Alexandra Wood, and Effy Vayena. 2018. "A Harm-Reduction Framework for Algorithmic Fairness”. IEEE Security \& Privacy, May/June 2018, pp. 34-45.

CMMI Product Team. 2010. CMMI® for Development. v. 1.3, Software Engineering Institute, Nov. 2010, http://cmmiinstitute.com/cmmi-models.

Fortinet. 2017. Ransomware Guide: Mapping the Ransomware Landscape. Fortinet Inc., Sunnyvale CA. http://www.fortinet.com.

Gotterbarn, Don, and Tony Clear. 2004. "Using SoDISTM as a Risk Analysis Process: A Teaching Perspective". Sixth Australasian Computing Education Conference (ACE2004). Dunedin, New Zealand. Conferences in Research and Practice in Information Technology. vol. 30, pp. 83-90.

Hartmann, Kim, and Keir Giles. 2018. "Net Neutrality in the Context of Cyber Warfare". 2018 10th International Conf. on Cyber Conflict. NATO CCD COE Publications, pp. 139-157.

Hoffman, K. E. 2017. "Permission denied: Mobile application security". SC Magazine. Haymarket Media Inc., New York NY, Feb 2017, pp. 24-27.

ISACA. 2015. CRISC® Review Manual 2015, ISACA, Arlington Heights IL USA.

ISACA. 2013. CISM® Review Manual 2013, ISACA, Arlington Heights IL USA, pp. 97-112.

Jaffee, L. 2017. “Always connected comes with risks”. SC Magazine, Haymarket Media Inc., New York NY, Feb. 2017, pp. 20-23.

Johnsen, A., G. Dodig Crnkovic, K. Lundqvist, K. H“anninen, and P. Pettersson. 2017. "Risk-based Decision-making Fallacies: Why Present Functional Safety Standards Are Not Enough". 2017 IEEE International Conference on Software Architecture Workshops. Pp. 153-160. 
Kahn, S. 1986. "Economic Estimates of the Value of Life”. IEEE Technology and Society Magazine. June, 1986.

Khalili, M. M., P. Naghizadeh, and M. Liu. 2018. "Designing Cyber Insurance Policies: Role of PreScreening and Security Interdependence". IEEE Transactions on Information Forensics and Security vol. 13, no. 9, March 2018.

KnowBe4. 2017. Report: The 2017 Endpoint Protection Ransomware Effectiveness Report. KnowBe4 Inc., Clearwater Florida. http://www.KnowBe4.com.

McAfee. 2018a. McAfee Mobile Threat Report Q1, 2018. Feb. 2018, McAfee Labs, Santa Clara, CA.

McAfee, 2018b. McAfee Labs Threats Report. Sept. 2018. McAfee Labs, Santa Clara, CA.

Meek, T., H. Burham, N. Beltaif, A. Kaadoor, T. Akhter. 2016. "Managing the Ethical and Risk Implications of Rapid Advances in Artificial Intelligence: A Literature Review". 2016 Proc. Of PICMET '16: Technology Management for Social Innovation. IEEE, pp. 682-693.

Ponemon. 2018. 2018 Cost of Data Breach Study: Global Overview. Ponemon Institute, July 2018.

Symantec. 2017. Internet Security Threat Report vol. 22. April 2017. Symantec Corp., Mountain View CA, 2018.

Symantec. 2018. Internet Security Threat Report vol. 23. Symantec Corp., Mountain View CA, 2018.

Verizon. 2018. 2018 Data Breach Investigations Report. 11th ed. Verizon, http://www.verizon.com.

\section{AUTHOR BIOGRAPHIES}

SUSAN J LINCKE is an Associate Professor of Computer Sciences at University of Wisconsin-Parkside. She holds an MS and PhD in Computer Science from Illinois Institute of Technology, and is CISA certified. She has extensive industry experience as a programmer analyst at GE, MCI, and Motorola. She is the author of Security Planning: An Applied Approach. Her email address lincke@uwp.edu.

MADHAVI ADAVI worked as a senior programmer analyst at Computer Science Corporation in India, before earning her MS Computer Information Systems, specializing in cyber-security, at the University of Wisconsin-Parkside in 2017. Since then, she has worked in the computer field in the Chicago and Milwaukee area. Her email address is himadhavi@gmail.com. 